exacerbating replication defects in tumour cells undergoing chemotherapy, and low levels indicating treatment resistance.

SAMHD1 is often mutated in leukaemias and solid tumours ${ }^{9,10}$. Although altered nucleotide levels might well perturb DNA replication and contribute to tumour development when SAMHD1 is mutated, Coquel and colleagues' research provides an alternative explanation. Fork-resection defects in SAMHD1-deficient cells might lead to increased problems with DNA replication - a phenomenon central to cancer development ${ }^{4,6}$.

It remains unclear whether cGAS-STING activity, such as that induced by SAMHD1 deficiency, promotes or prevents tumour formation. On one hand, increased levels of cytoplasmic nucleic acids and cGAS-STING activation can signal potentially dangerous replication problems in abnormal cells, and thereby promote their elimination by the immune system ${ }^{6}$. Indeed, cGAS-STING immune signalling is suppressed in some cancers. On the other hand, persistent cGASSTING signalling can lead to a chronic proinflammatory response, which can promote tumour development and spread ${ }^{11,12}$. Further work is required to better understand the role of these immune responses in cancer.

Coquel et al. also advance our understanding of the causes of immune disease by shedding light on the important question of whether the different mutations associated with AicardiGoutières syndrome promote disease through a common mechanism. Although cells lacking SAMHD1, TREX1 and RNase H2 all trigger interferon responses through cytoplasmic cGAS-STING signalling, there is some evidence that the activators of this pathway might be distinct. For SAMHD1 and TREX1, there are now links to ssDNA produced during DNA replication ${ }^{1,3}$, but a role for other cytoplasmic nucleic acids is yet to be ruled out. For RNase H2, cGAS-STING is induced by DNA derived from micronuclei ${ }^{13}$ - small, aberrant nuclei that form when chromosomes fail to segregate properly into sister cells during cell division. Although double-stranded DNA can activate cGAS-STING, ssDNA might also be present in micronuclei and thus contribute to activation of the pathway. It will be interesting to further define exactly which nucleic acids drive this syndrome.

Finally, it remains unclear how nucleic acids are released into the cytoplasm to activate the cGAS-STING pathway. One possibility is that they escape the nucleus after the surrounding nuclear envelope breaks down during cell division ${ }^{13}$. However, Coquel et al. found that cytoplasmic ssDNA accumulates rapidly in SAMHD1-deficient cells, even before division, suggesting that other pathways are involved. Understanding how the pathological build-up of nucleic acids in the cytoplasm of cells occurs might help us to identify molecular targets that have the potential to be therapeutically manipulated in immune disease.
Madzia P. Crossley and Karlene A. Cimprich are in the Department of Chemical \& Systems Biology, Stanford University, Stanford,

California 94305, USA.

e-mails: cimprich@stanford.edu; mpcross@stanford.edu

1. Coquel, F. et al. Nature 557, 57-61 (2018).

2. Goldstone, D. C. et al. Nature $480,379-382$ (2011)

3. Crow, Y. J. \& Manel, A. Nature Rev. Immunol. 15, 429-440 (2015)

4. Zeman, M. K. \& Cimprich, K. A. Nature Cell Biol. 16 2-9 (2014).
5. Franzolin, E. et al. Proc. Natl Acad. Sci. USA 110 , 14272-14277 (2013)

6. Pasero, P. \& Vindigni, A. Ann. Rev. Genet. $\mathbf{5 1}$ 477-499 (2017).

7. Schlacher, K. et al. Cell 145, 529-542 (2011)

8. Chaudhuri, A. R. et al. Nature $535,382-387$ (2016).

9. Clifford, R. et al. Blood 123, 1021-1031 (2014).

10. Rentoft, M. et al. Proc. Natl Acad. Sci. USA 113. 4723-4728 (2016)

11.Dou, Z. et al. Nature 550, 402-406 (2017).

12.Bakhoum, S. F. et al. Nature 553, 467-472 (2018).

13. Mackenzie, K. J. et al. Nature 548, 461-465 (2017).

\title{
ASTRONOMY
}

\section{Helium discovered in the tail of an exoplanet}

As the exoplanet WASP-107b orbits its host star, its atmosphere escapes to form a comet-like tail. Helium atoms detected in the escaping gases give astronomers a powerful tool for investigating exoplanetary atmospheres. SEE LETTER P. 68

\section{DRAKE DEMING}

$\mathrm{H}$ elium is ubiquitous in the Universe. Large amounts were generated in the Big Bang ${ }^{1}$, and nearly every star begins its life by producing helium in its core through the nuclear fusion of hydrogen. The atmospheres of giant exoplanets are expected to have an abundance of helium ${ }^{2}$, because these planets formed from recycled gas and dust from a previous generation of stars. However, searches for helium in such atmospheres have been unsuccessful ${ }^{3}$. On page 68, Spake et al. ${ }^{4}$

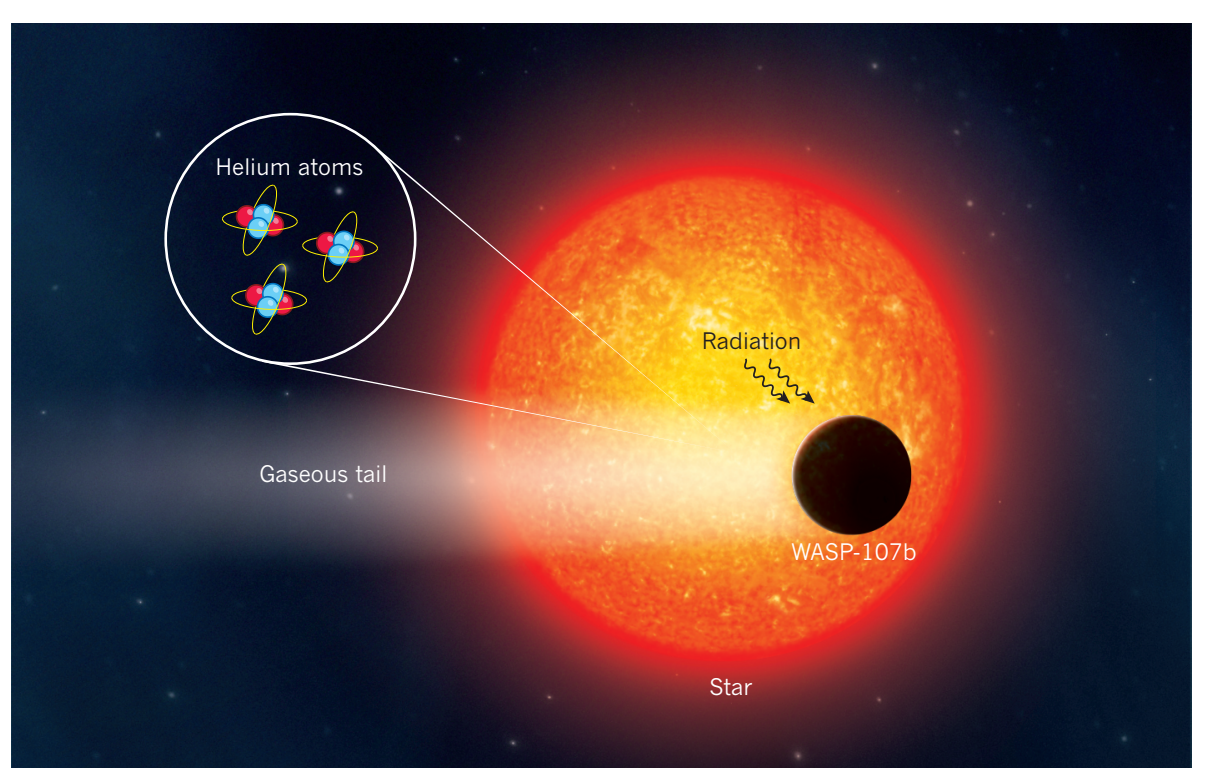

Figure 1 | The escaping atmosphere of WASP-107b. As the giant exoplanet WASP-107b orbits its host star, ultraviolet radiation from the star energizes the planet's atmosphere. Spake et al. ${ }^{4}$ show that this causes the atmosphere to escape, and to form a gaseous tail. The authors detected helium atoms in the escaping gases. This is the first time helium has been identified in an exoplanetary atmosphere. report the discovery of helium atoms in the eroding atmosphere of the giant exoplanet WASP-107b. Their work opens a new chapter in the study of exoplanetary atmospheres.

WASP-107b is of comparable size to Jupiter, but has about one-eighth the mass. The exoplanet's low mass relative to its substantial size makes it difficult for the planet to retain its atmosphere - especially in the presence of strong ultraviolet radiation from its host star. Although this star is smaller and cooler than the Sun, it is threaded with magnetic fields produced by the star. Contortions of these 
fields emit ultraviolet radiation that energizes the planet's atmosphere.

Spake et al. observed WASP-107b using a camera on board the Hubble Space Telescope, and concluded that the planet's atmosphere escapes to form a comet-like tail (Fig. 1). Astronomers have long known that giant planets can lose their atmospheres in this fashion ${ }^{5}$, so this aspect of Spake and colleagues' work is not surprising. But the authors have added a key twist to the story. Until now, only hydrogen (the main component of giant planets) and a few elements with low abundances ${ }^{6}$ have been identified in eroding exoplanetary atmospheres.

Atoms in the gaseous tail of an exoplanet are most easily detected when they absorb stellar light during a transit - a passage of the planet in front of its host star. However, atoms in such a tenuous tail have a tendency to relax to their lowest-energy (ground) state. In this state, most atoms absorb mainly ultraviolet light, and measuring such absorption is difficult for two reasons.

First, Earth's atmosphere is opaque to most ultraviolet light, which means that absorption measurements must be made from space. Currently, only Hubble has the capability for ultraviolet studies of exoplanetary atmospheres, and this telescope could reach the end of its mission lifetime in the next decade. Second, the pattern of how much ultraviolet stellar light is absorbed by transiting planets as a function of time or wavelength tends to be complex. Such complexity makes it difficult to interpret ultraviolet measurements of a transiting planet's atmosphere.

Fortunately, helium atoms have a long-lived (metastable) state, in addition to the ground state. Metastable helium atoms absorb nearinfrared stellar light, which has a wavelength only slightly beyond the limits of human vision. Measurements at this wavelength are much easier to interpret than those at ultraviolet wavelengths.

Spake and colleagues observed a transit of WASP-107b, and measured the amount of near-infrared stellar light that was transmitted through the planet's eroding atmosphere as a function of wavelength. The authors identified a narrow absorption feature that they associated with metastable helium atoms (see Fig. 1 of the paper ${ }^{4}$ ). This signal is more than five times greater than any false signal that could be produced by stellar activity.

Detecting helium in the escaping atmospheres of other exoplanets will be difficult because the absorption signal is intrinsically weak, especially for planets smaller than WASP-107b. However, astronomers will eagerly rise to the challenge. The near-infrared signature of metastable helium is readily transmitted through Earth's atmosphere, which means that eroding exoplanetary atmospheres could be probed using ground-based telescopes. The advent of a new generation of extremely large telescopes at ground-based observatories $^{7}$ will allow astronomers to study the escaping atmospheres of planets as small as Neptune, which has a radius four times that of Earth.

Theorists have predicted that the atmospheres of Neptune-sized exoplanets could be rich in helium ${ }^{8}$, owing to differences in the rates at which hydrogen and helium are lost to space. Like other giant planets, these bodies are thought to start out with atmospheres of predominantly hydrogen, abundant helium and smaller amounts of elements heavier than helium. As their atmospheres escape, hydrogen is lost fastest, leading to a gradual relative enrichment in the helium content of the atmosphere.

Heavier elements such as carbon and oxygen would be slow to escape, and could in principle be present in exoplanetary atmospheres in concentrated amounts. These heavier elements are key to understanding both how planets form and how they acquire their atmospheres. For planetary astronomers, an escaping atmosphere that is rich in heavy elements is something of a cosmic treasure, providing ample scientific opportunities to study planetary formation and evolution. Spake and colleagues' detection of helium in WASP-107b will enable astronomers to look for atmospheres that are rich in helium, and perhaps in heavier elements, thereby opening a new subfield of exoplanetary science.

Drake Deming is in the Department of Astronomy, University of Maryland, College Park, Maryland 20742, USA.

e-mail:ddeming@astro.umd.edu

1. Alpher, R. A., Bethe, H. \& Gamow, G. Phys. Rev. 73, 803-804 (1948).

2. Seager, S. \& Sasselov, D. D. Astrophys. J. 537 916-921 (2000).

3. Moutou, C., Coustenis, A., Schneider, J., Queloz, D. \& Mayor, M. Astron. Astrophys. 405, 341-348 (2003).

4. Spake, J. J. et al. Nature 557, 68-70 (2018)

5. Vidal-Madjar, A. et al. Nature 422, 143-146 (2003)

6. Ben-Jaffel, L. \& Ballester, G. E. Astron. Astrophys. 553, A52 (2013).

7. Liske, J., Padovani, P. \& Kissler-Patig, M. Proc. SPIE 8444, 84441I (2012)

8. Hu, R., Seager, S. \& Yung, Y. L. Astrophys. J. 807, 8 (2015).

\section{PALAEONTOLOGY}

\section{Evolutionary insights from an ancient bird}

\section{Ichthyornis dispar is a key extinct bird species from when birds were shedding characteristics of their dinosaur ancestors and evolving their current features. A reconstructed skull of I. dispar now illuminates this transition. SEE LETTER P.96}

\section{KEVIN PADIAN}

$\mathrm{T}$ The distinctive features of birds, from beaks to feathers, provide a stark separation between avians and other animal groups. But how did the features of the bird skull evolve? On page 96, Field et al. ${ }^{1}$ present a computerized reconstruction of the skull of a pivotal early bird that brings avian evolution into sharper focus.

In the late 1800s, the palaeontologist Othniel C. Marsh and his field crews made many of the first reported discoveries of ancient dinosaurs and mammals from western North America, amassing a fossilized 'bestiary' that dwarfed what was then known from Europe ${ }^{2}$. Marsh's treasures were constantly in the headlines, perhaps never more so than when he published ${ }^{3}$ his monograph Odontornithes in 1880, which reported several previously undescribed fossil birds of the mid-Cretaceous period (around 80 million to 87 million years ago) from the shores of Kansas and nearby states. Familiar yet strange in many ways, these creatures were so archaic that they retained teeth and substantial bony tails, thus providing clues to the reptilian origin of birds. When Charles Darwin received a copy of the monograph from Marsh, the letter that he wrote back to Marsh said: "Your work on these old birds and on the many fossil animals of N. America has afforded the best support to the theory of evolution, which has appeared within the last 20 years" (see go.nature.com/2hhjxrd).

The specimens Marsh presented in Odontornithes were predominantly from two contrasting bird genera: Hesperornis, which was flightless and essentially wingless, standing 1.3-1.8 metres tall and comparable to today's loons, and a tern-like bird called Ichthyornis, which had an average wingspan of about 60 centimetres (ref. 3). However, neither was closely related to living loons or terns. Both birds had many sharp, curved teeth, which were absent only from the front part of the upper jaw, and their beaks were covered by a horny sheath. Unfortunately, the excavated bones, being small, fragile and of an elaborate architecture, were badly crushed, and proved challenging to prepare. The restoration, mounting and illustration of the specimens were, shall we say, somewhat overenthusiastic. The specimens could be convincingly described only after the mounts had been 